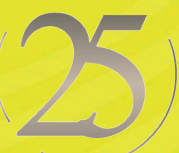

ANMERSARO
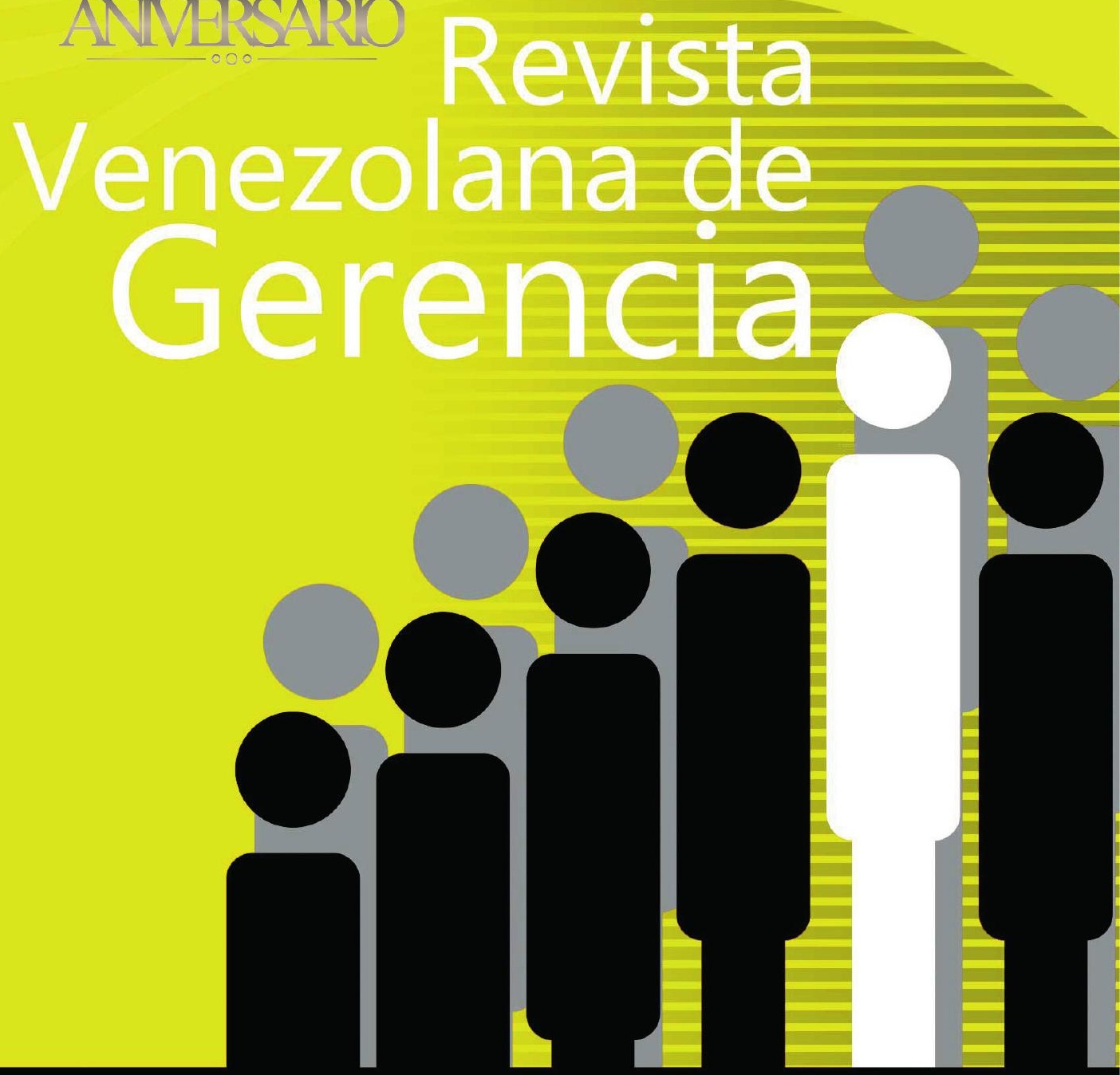


\title{
Competencias digitales en directivos y profesores en el contexto de educación remota del año 2020
}

\author{
Holguin-Alvarez, Jhon* \\ Apaza-Quispe, Juan* \\ Ruiz Salazar, Jenny Maria \\ Picoy Gonzales, Juan Antonio ${ }^{* * *}$
}

\section{Resumen}

Las competencias digitales son parte del perfil del profesional educativo en situaciones de virtualidad. Se asume mejor desarrollo de estas capacidades en los sistemas de educación virtual emergentes en razón de los modelos educativos surgidos en pandemia. El objetivo de la investigación fue analizar las competencias digitales de la educación remota de 280 directivos y docentes de dos contextos situacionales. Mediante el enfoque cuantitativo, se realizó un estudio de nivel descriptivo, con método de evaluación transeccional. Ello permitió la aplicación de una escala de competencias digitales en dos períodos del año de prevalencia pandémica por Sars-Cov-2 en el año 2020. Como resultados se encontraron diferencias en el promedio e índices analizados, estos sustentaron el incremento de los niveles de competencias digitales en docentes. Con respecto a los directivos de instituciones educativas ubicadas en contextos vulnerables, se encontraron mayores niveles y promedios en las capacidades de adquisición y uso de la información, comunicación y colaboración, y en la creación de recursos digitales. Como conclusión, existe mayor nivel de competencias digitales en profesores que en los directivos, destacando mayores niveles en las capacidades de comunicación y colaboración, así como también en la creación de recursos digitales.

Palabras clave: Aislamiento Social; Competencias Digitales; Educación Remota; Formación Docente; Formación de Directivos.

\section{Recibido: $20.01 .21 \quad$ Aceptado: 25.03 .21}

Docente del Instituto de Investigación. Especialidad de Educación Primaria. Universidad César Vallejo, Perú. Correspondencia: jholguin@ucv.edu.pe, hhonholguinalvarez@gmail.com. ORCID ID: https://orcid.org/0000-0001-5786-0763

* Docente de la Escuela Profesional de Arte y Diseño Gráfico Empresarial. Universidad César Vallejo, Perú. ORCID ID: https://orcid.org/0000-0002-1157-7185

*** Docente del área de Ciencias. Universidad Tecnológica del Perú. ORCID ID: https://orcid. org/0000-0001-9882-3133

*to** Docente de la Facultad de Ciencias de la Salud, Escuela Profesional de Obstetricia. Universidad Nacional de Huancavelica. ORCID ID: https://orcid.org/0000-0003-0372-6328 


\title{
Digital Competences in Managers and Isolated Teachers in the Context of Remote Education in 2020
}

\begin{abstract}
Digital competences are part of the profile of the educational professional in virtual situations. Better development of these capacities is assumed in emerging virtual education systems due to the educational models that emerged in a pandemic. The objective of the research was to analyze the digital competences in remote education of 280 managers and teachers from two situational contexts. Using the quantitative approach, a descriptive study was carried out, with a transectional evaluation method. This allowed the application of a scale of digital competences in two periods of the year of pandemic prevalence by Sars-Cov-2 in the year 2020. As results, differences were found in the average and indices of difference analyzed, these sustained the increase in levels of digital competences in teachers, unlike the directors of educational institutions located in vulnerable contexts, higher levels and averages were found in the abilities of acquisition and use of information, communication and collaboration, and in the creation of digital resources. As a conclusion, there is a higher level of digital competences developed in teachers as opposed to managers, highlighting higher levels of communication and collaboration skills, as well as the creation of digital resources.
\end{abstract}

Key Words: Digital Competences; Management Training; Remote Education; Social isolation; Teacher Training.

\section{Introducción}

Durante el año 2020, la mortalidad de infección por Sars-CoV-2 (Covid-19) originó un aproximado de dos millones de muertes humanas, y más de 100 millones de personas infectadas en el mundo. En el Perú, el índice de muertes se incrementó de 300 en abril a 37.724 personas en diciembre, siendo el rango de mayor prevalencia de infección entre los meses de mayo y agosto (comunicados $\mathrm{N}^{\circ}$. 68 / N $\mathrm{N}^{\circ}$. 375, Ministerio de Salud, 2020). En cuanto a contagios, la frecuencia se incrementó de 13.489 en el mes de abril a 101.799 en el mes de diciembre del año 2020 (comunicados $\mathrm{N}^{\circ}$. $68 / \mathrm{N}^{\circ}$. 375, Minsa, 2020). Este panorama, dio inicio al descubrirse el primer caso de infección de dicha enfermedad en el mes de marzo, lo cual generó esfuerzos por parte del gobierno para mitigar sus efectos en la población.

Mediante el Decreto Supremo $\mathrm{N}^{\circ}$. 046-2020-PCM (Gobierno Nacional del Perú, 2020) el gobierno nacional decretó el aislamiento social obligatorio para menguar la emergencia sanitaria en el territorio nacional, apareciendo medidas homólogas en el sector educativo. Entre 
las más importantes fueron la 046-2020PCM, mediante la cual se implementó y ejecutó el sistema de educación remota mediante el programa Aprendo en Casa, dirigido a todos los niveles de Educación Básica Regular (Ministerio de Educación del Perú, 2020); cuyos contenidos se transmitieron a nivel nacional por medios televisivos, radiales y de Internet.

En ese sentido, las normativas se plantearon para que los docentes ejecuten tres tareas esenciales: a) retroalimentación formativa, b) seguimiento académico y tutorial, c) evaluación continua. Ello exigió que el profesorado se adaptara al uso de la plataforma pública a la par de otras de tipo virtual mediante el uso de computadores personales. Muchos docentes terminaron utilizando sus dispositivos móviles [celulares] para realizar llamadas y realizar las tres tareas [a, b, c]. Adicional a ello se encontró: baja calidad de datos de Internet, cableado y desconocimiento en el uso de las plataformas virtuales de aprendizaje.

La adaptación fue paradójica, pero demostró una realidad innegable. Se supo que hasta antes de la pandemia casi $20 \%$ de adultos mayores accede a alguna herramienta digital en el Perú, es así que, las personas con edad menor a 50 años, lo hacen en mayor porcentaje, siendo parte de esta población, el profesorado de las escuelas públicas, y el otro, algún directivo de las mismas (Instituto Nacional de Estadística e Informática del Perú [INEI], 2019). Cabe señalar que, más del $95 \%$ de los directores de las escuelas peruanas utilizaron recursos tecnológicos antes del año 2020 (INEI, 2019). Aunque, fuera del trabajo, alrededor del $30 \%$ de pobladores tiene problemas de fluidez de datos de Internet (INEI, 2020), teniendo en cuenta esta fuente, cabe estimar que alguna parte de esa población es directivo o profesor en el país.

En líneas generales, los problemas de práctica, uso de recursos e interacción de los directivos y el profesorado redundaron en la gestión directiva y en la gestión pedagógica durante el transcurso de la pandemia en el año 2020, acentuándose el problema en el dominio de la información y en su uso como recurso muy requerido para la educación remota, tanto en la ciudad de Lima como en otras regiones de Latinoamérica, a pesar que desde al año 2019, el Banco Mundial declaró que las competencias digitales deben ser intrínsecas en la formación del ciudadano digital (World Bank, 2019), por ende el profesorado está inmerso en este grupo como parte de la adaptación a los entornos de vida virtual.

Engen (2019) encuentra queen la población docente existe gran motivación para capacitarse en estrategias complementarias para la enseñanza, también existe cierta cultura arraigada para adaptarse a algún tipo de educación virtual como por ejemplo, el E-learning o el M-learning, tomando en cuenta que en dicho proceso adaptativo, se logra la capacitación de sus habilidades digitales. Por otro lado, otros autores han indicado que este problema suele ocurrir desde la etapa formativa, en cuyos medios para aprender se utilizan pocas estrategias colaborativas (Garzón et al, 2020, Engen, 2019). Más aún, se sabe que el alumnado se culturiza mediante el uso de dispositivos con alto nivel de acceso informativo, por ende, suele interactuar en mayor medida en esas tecnologías (Aristizabal \& Cruz, 2018).

Estas primeras evidencias permiten cuestionar si los procesos formativos en la educación virtual, ocurren en la interacción dentro de la 
Competencias digitales en directivos y profesores aislados en el contexto de educación remota del año 2020

escuela. Entonces, ¿las situaciones emergentes como la actual generan interacciones de desarrollo mutuo entre los participantes que compiten por desarrollarse profesionalmente?, por cuanto, cabe averiguar el desarrollo de las competencias digitales durante el evento pandémico, a un año de su prevalencia en la población peruana. En este sentido, el objetivo del presente trabajo se concreta en analizar las competencias digitales de directivos y profesores de las instituciones educativas públicas y privadas de tres distritos de Lima, precisando como período los inicios de la pandemia y finales del año 2020. Para este caso, se optó por la metodología cuantitativa, descriptiva de tipo transeccional.

\section{Competencias Digitales: Enfoques teóricos}

El proceso de interrelaciones humana se entiende desde distintas perspectivas comunicacionales, entre ellas, el conectivismo y el construccionismo digital; estos representan las primeras evidencias del aprendizaje mediante el uso y dominio de la información obtenida de las redes (Siemens, 2004; Teixeira \& Martini, 2019).

En la educación y formación de profesores y directivos, desde el plano de la virtualidad, se puede entender al conectivismo como un enfoque teórico, que basa los procesos de aprendizaje en la búsqueda, uso de información y los medios para generar nodos de culturización en la comunidad digital. Desde el construccionismo digital, se comprende que los sistemas de nodos generados por los aprendices en la interacción virtual, permiten obtener información de distinta índole (Lasso et al, 2017).

Sin embargo, esta perspectiva exige que en la culturización, se provoquen procesos adaptativos de enseñanza y aprendizaje por retroalimentación; considerando las competencias digitales como medio de aprendizaje para obtener la información, pero más aún, para emprender procesos formativos mediante el uso de diversas competencias como la comunicación, la crítica, la reflexión, el análisis y la investigación. Los directivos y profesores de instituciones educativas, buscan desarrollar estos procesos, desplegados desde la gestión de instituciones educativas inteligentes, dominadas por algún tipo de gerencia o liderazgo organizacional.

Las competencias digitales se han comprendido como capacidades vitales para convivir en espacios virtuales. Desde la educación, las competencias digitales son capacidades para generar procesos de enseñanza (Garzón et al, 2020; Girón-Escudero et al, 2019); aunque, las perspectivas formativas basadas en la autopercepción del desempeño educativo las establecen como las destrezas para conocer y utilizar la información para generar procesos de enseñanza (Brauer, 2019; Brolpito, 2018; Tourón et al, 2018). En este sentido, es necesaria la inclusión del aspecto actitudinal, el cual unido a los conocimientos del usuario, permite generar otros conocimientos en los receptores del aprendizaje con cierta destreza (Janssen et al, 2013). Estas destrezas deben provocar aprendizajes retroalimentados entre el docente y el alumno, entre el profesor y el directivo. Las perspectivas formativas de la comunicación de la información sugieren generar retroalimentación, aunque ese proceso implica la capacidad para 
colaborar con el entorno.

Allueva \& Alejandre (2019) y Ruíz-Velazco \& Barcena (2019) han establecido destrezas más específicas como indicadores incluidos de forma conexa a cada dimensión de estas competencias, entre las que resaltan, la colaboración, asociación e independencia para aprendizaje. En este sentido, se acuerda que aprendices de enseñanza transforman la información (Spante et al, 2018), la implementan en actividades estructurales y las aplican en contextos de aprendizaje interactivos, donde se utilizan capacidades biológicas aplicadas al conectivismo como: a) conversación, b) discusión, c) participación, d) silencio. Algunas evidencias las han planteado en el sistema de NETiquetas implementadas en los entornos virtuales de aprendizaje, aunque sean moderadas, estas se utilizan para lograr relaciones interpersonales entre los aprendices.

Aunque son escasos los planteamientos que indiquen que el aprendizaje asociativo se genere mediante destrezas formativas pedagógicas en espacios virtuales, se ha creído que las competencias digitales son medios para la alfabetización virtual (Fallon, 2020), por ello, es necesario entender que las competencias digitales son útiles en el marco de enseñanza asociativa; se utilizan diversos medios tecnológicos considerados elementos virtuales de aprendizaje externos o internos a las plataformas de virtualización, permitiendo aprendizajes, sin ser únicamente útiles para la socialización.

En cuanto a las dimensiones, Tourón et al, (2018), determinaron cinco aspectos en que se utiliza la información, desde la perspectiva educativa. Entre estas dimensiones se genera: el uso de la información acompañado del conocimiento, la habilidad para comunicarse y colaborar en el aprendizaje, crear el contenido digital para su utilidad, aplicación de acciones de seguridad mediática, analizar y resolver problemas en los entornos de aprendizaje. Respecto a la dimensión uso de la información, los profesionales de la educación se basan en el uso de las habilidades para conseguir, organizar, analizar y utilizar la información como recurso intangible para la generación del conocimiento, así como provocar el uso de las capacidades en los estudiantes.

Respecto a la capacidad para la comunicación y colaboración, se requiere desarrollar, en los estudiantes, distintas capacidades como la búsqueda de la interacción social, la colaboración como medio aptitudinal en el dominio de la información; y las actitudes reflexivas sobre el contenido que investigan. En relación a la creación del contenido digital, el profesorado debe utilizar los recursos para generar actividades mediáticas en el entorno virtual de aprendizaje, por lo que se asume que los docentes aplican el uso de elementos de redes o del Internet [audios, vídeos, Papers, entre otros].

De acuerdo a Tourón et al, (2018), la dimensión aplicación de la seguridad mediática es la habilidad que usualmente los docentes tienden a utilizar muy poco respecto a las actividades virtuales, por lo cual, esta debe permitir que los estudiantes interactúen de forma segura en los espacios en que se introducen para convivir en comunidades digitales, o en espacios para conseguir información en su formación académica. Aunado a esto, la competencia para resolver problemas también debe acompañar a esta capacidad, puesto que en las interrelaciones de trabajo o de 
Competencias digitales en directivos y profesores aislados en el contexto de educación remota del año 2020

socialización, los profesores necesitan de aptitudes para aplicar los recursos, activando los mecanismos necesarios para plantear soluciones a los problemas emergentes antes, durante o después del desarrollo virtual de aprendizaje, aunque en muchos casos suelen aparecer durante el ejercicio de las sesiones de clase $o$ actividades de aprendizaje.

\section{Caracterización de los participantes y desarrollo de la investigación}

La investigación se enfoca en el método positivista, el tipo de estudio es no experimental. El nivel es descriptivo transeccional, ya que se busca indagar en las competencias digitales de los directivos y de los docentes que laboran en las instituciones educativas del país de Perú. De igual modo, se desarrollan mecanismos de comparación estadística para deslindar competencias digitales con determinado nivel en razón de variables como la pobreza, capacitación y grupo etario. En cuanto a la muestra de estudio, se trabajó con 280 individuos involucrados en diez instituciones educativas de la ciudad de Lima (directores $y$ subdirectores $_{(n)}=80 ;$ docentes $\left._{(n)}=200\right)$, todas correspondientes a los distritos: Comas, Puente Piedra y Los Olivos. La edad promedio presentó diferencias leves en cuanto al cargo (directores $y$ subdirectores $=54,5$ años; docentes $=$ 41,2 años). En la tabla 1, se describe la distribución por distrito y edad.

Tabla 1

Frecuencia y distribución de directivos y docentes por distrito, contexto y edad.

\begin{tabular}{lllll}
\hline Distritos & Contexto & Función & (f) & \multicolumn{1}{l}{$\begin{array}{l}\mathrm{e} \\
\text { (años, } \\
\text { mes) }\end{array}$} \\
\hline Comas & $\begin{array}{l}\text { Vulnerable } \\
{[n=115]}\end{array}$ & Directivos & 35 & 49,3 \\
\cline { 3 - 5 } & Docentes & 80 & 40,5 \\
\hline $\begin{array}{l}\text { Puente } \\
\text { Piedra }\end{array}$ & $\begin{array}{l}\text { Media- } \\
\text { namente } \\
\text { vulnerable } \\
\text { Los Olivos }\end{array}$ & Directivos & 25 & 57,6 \\
\cline { 3 - 5 } & Docentes & 60 & 38,9 \\
\cline { 3 - 5 } & Directivos & 20 & 52,2 \\
\cline { 3 - 5 } & Docentes & 60 & 44,2 \\
\hline
\end{tabular}

Fuente: Elaboración propia

Nota: $(f)$ = frecuencia; $(e)=$ edad .

Como se puede observar en la tabla 1 , se tuvo mayor acceso a instituciones educativas en contextos medianamente vulnerables, las cuales colaboraron en mayor número con las encuestas realizadas. Respecto al género, la distribución presentó mayor número de

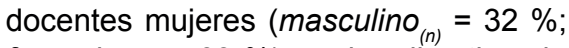
femenino $_{(n)}=68 \%$ ), en los directivos la diferencia fue menor (masculino $_{(n)}=56$ 
$\%$, femenino ${ }_{(\mathrm{n})}=44 \%$ ). Es importante declarar que en el rubro de directivos también se incluyeron a los subdirectores quienes lo aceptaron anticipadamente.

Por otro lado, es necesario mencionar que los docentes ejecutaban su tarea académica durante el mes de abril hasta el mes de diciembre de 2020 cuando se ejecutó el recojo de datos, por lo cual, el acceso al total de instituciones, como parte de la población, presentó distintas dificultades relativas a la red de Internet, en algunos casos muchos docentes no contaban con datos disponibles para responder los formularios de evaluación, ya que el contacto personal se debía evitar por medidas de bioseguridad.

En cuanto al instrumento de evaluación, se utilizó el Cuestionario de Competencia Digital Docente [CCDD] de Tourón et al, (2018), el cual se constituyó por 31 proposiciones (ítems) a los cuales se adaptó cuatro opciones de respuesta: (1) completamente, (2) dominio básico, (3) dominio regular, (4) ningún dominio. Las proposiciones medían el conocimiento y uso sobre recursos y utilidades en el plano de la práctica pedagógica. Se adecuó el instrumento para ayudar a que los profesores completasen el cuestionario. La aplicación constó de dos pruebas piloto para cada instrumento, así también, este proceso se acompañó con una evaluación por pares cruzados para revisar la estructura de las dimensiones e ítems integrados.

En este último paso, se evaluó la opinión de pares con cinco jueces expertos e investigadores en psicología educacional, educación universitaria y escolar. La fiabilidad presentó índices de correlación significativos en el constructo competencia digital, y en sus

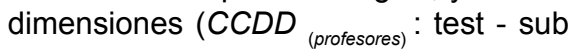

test ${ }_{(\text {rango } r)}=, 831-, 898 ;$ ítem - test ${ }_{(\text {rango }}$ r) $=, 823-, 891 ; p<.005 ; C C D D_{\text {(directivos) }}$ : test - sub test ${ }_{(\text {rango } r)}=, 901-, 926$; ítem - test $\left.{ }_{(\text {rango } r)}=, 921-, 927 ; p<.005\right)$. Se calculó el índice Alfa de Cronbach para analizar la consistencia interna por cada instrumento, siendo estos aceptables para el estudio. Respecto a la validación por expertos, ambos instrumentos alcanzaron una aceptación mayor a 95 $\%$.

Respecto al procedimiento de evaluación de los individuos de esta muestra, esta se realizó por pasos estructurados en medios de comunicación virtual. Como primer paso, se contactó a los directores y subdirectores de todas las instituciones educativas mediante correo electrónico con una invitación al estudio desde el mes de marzo. Estas invitaciones se hicieron de forma masiva y repetitiva, ya que asumimos que dicha población evitaba participar debido a otros factores como, desesperación, incertidumbre, así también, por los cambios entre la educación presencial y la educación remota.

El proceso de adaptación se siguió hasta conseguir la respuesta de más de 50 directivos, los cuales se iban sumando a la aceptación de otros. Los docentes evaluados llegaron a ser 200 en su totalidad. Comenzamos a contactar a los participantes de la muestra desde el mes de abril, y se aplicó durante dos lapsos bimensuales de evaluación, aplicando la escala en los meses de mayo y Junio de 2020, y en el mes de noviembre y diciembre de 2020.

Respecto a los instrumentos, se sistematizaron en formularios Google, con el fin de utilizar los enlaces para compartirlos en medios virtuales de rápido acceso. A su vez, los datos recogidos mediante estos enlaces se 
Competencias digitales en directivos y profesores aislados en el contexto de educación remota del año 2020

registraban en una base de datos Excel de forma automática. Los enlaces fueron distribuidos a los profesores y directores mediante comunicaciones vía mensajes de WhatsApp y correos masivos. Cada instrumento incluyó un formulario breve con la solicitud del consentimiento informado, mediante el cual, cada individuo otorgó el uso de los datos brindados para su análisis y reporte. Cabe señalar que, se aplicó el criterio de anonimato respecto a la información de cada sujeto de estudio y de cada institución educativa implicada.

\section{Antecedentes en el estudio de las competencias digitales}

Los resultados sobre competencias digitales revelan que el problema de su utilización didáctica, radica en su práctica como una competencia vital en el profesorado del Perú. En principio, uno de los principales problemas es la falta de presupuesto económico para suplir las necesidades formativas de los docentes, siendo pocas las instituciones que generan la formación de sus empleados, por otro lado, los recursos tecnológicos tienden a ser poco modernos, limitándose solo al uso de computadoras personales; finalmente, existe bajo presupuesto para adquirir plataformas virtuales de aprendizaje que permitan a los profesores trabajar mediante otras modalidades como la educación semi-presencial $\mathrm{o}$ el aprendizaje guiado en casa.

Es importante acotar que los efectos del aislamiento y la educación remota emergente han provocado en los directivos la adaptación al modelo de gestión virtual de forma intempestiva. Al respecto, las evidencias reflejan similares dificultades para el profesorado que se capacita (Engen, 2019), haciéndolo en estrategias presenciales algunas orientadas al uso de recursos didácticos. En este caso, la pandemia ha exigido utilizar recursos, dispositivos y entornos virtuales de aprendizaje. Las dificultades son similares al contexto nacional, centrándose en que los profesores tienen problemas para utilizar tecnologías (Garzón et al, 2020), se deduce que también ocurre en los directivos.

Esto puede ser influyente en las poblaciones que dirigen los docentes y directivos en contextos con distintas necesidades, en especial necesidades económicas y de formación social como ha ocurrido hasta ahora en los contextos vulnerables. En sectores medianamente pobres, se encontró que los receptores de la enseñanza [sean directivos, profesores o alumnos], suelen culturizarse, adoptar hábitos comunicativos de acuerdo a las preferencias y gustos de ellos mismos, debido a que en la actividad de enseñanza se generan intercambios de conocimiento, información y afectividades (Aristizabal \& Cruz, 2018; Choudrie et al, 2018).

En este sentido, las tecnologías permiten a los colaboradores generar entornos de interrelación en paralelo a los procesos de asociación cognitiva, puesto que las competencias digitales aportan en el crecimiento socio-digital. En otros estudios, se encontró que los propósitos, el acceso y las guías [rutas] para aprender se pueden generar en estas interrelaciones, gamificar el aprendizaje como práctica aporta en ello debido a la motivación que generan los espacios para este fin (Andía et al, 2020; Sandí \& Sanz, 2020). En muchos casos, la pedagogía ejercida de este modo se pierde, las oportunidades en los aprendices se acortan, así también, los beneficios comienzan a desaparecer 
cuando los docentes presentan falta de dominio de los entornos al ejecutar sus clases.

Por lo anterior, el currículo actual necesita empoderarse de las necesidades que atañen al ser humano como ciudadano que vive y se comunica de forma segura (Tomczyk, 2020); pero, con mayor plenitud, las organizaciones educativas necesitan implementar este currículo con perspectiva formativa del ser humano que aprenda y, sobre todo, que aprenda a enseñar en la interacción digital con otros ciudadanos digitales.

En países centroamericanos como en Honduras, Acevedo-Duque et al, (2020), reportan que la adaptación de los docentes ha sido exigente durante el tiempo de pandemia, puesto que de una muestra de 353 profesores universitarios, casi el $15 \%$ desarrollaba actividades sincrónicas y asincrónicas virtuales de forma regular o poco frecuente; por otro lado, $27 \%$ lo realizaba de forma frecuente. En el contexto de Perú, Holguin-Álvarez et al, (2020), encontraron que las competencias digitales se diferencian respecto al perfil del contrato docente, por cuanto, en la pandemia, los profesores con nombramiento docente desarrollaron mejores competencias (más del 80 $\%$ ), que aquellos con contrato único. En este caso, las oportunidades de la carrera pública magisterial han exigido más desarrollo de estas competencias, adaptadas a la educación en la emergencia sanitaria.

Lo señalado puede describirse gracias a los aportes de Levano-Francia et al, (2019), quienes describen que la adaptación hacia las nuevas exigencias de desarrollo profesional se debe a los avances tecnológicos los cuales, usualmente, los docentes se encuentran cada vez más obligados a hacerlo. De esto se desliga que la necesidad puede haber hecho que las competencias digitales se incrementasen más en la pandemia.

\section{Competencias digitales en docentes de Educación Básica.}

El análisis de distribución de normalidad, presentó datos no significativos, por cuanto existió bondad de normalidad de ajustes tanto para los datos sobre directivos como en profesores (Dist. (directivos, profesores) $p>.005$ ). Por cuanto, se aplicaron pruebas paramétricas para contrastar los datos relacionados de las competencias digitales en el inicio de la pandemia en Perú (mayo y Junio de 2020) y los datos relativos al proceso de prevalencia del Covid-19 (noviembre y diciembre de 2020).

\subsection{Competencias digitales de acuerdo al contexto}

En cuanto al análisis general de la muestra [directores y profesores], se encontró diferencias marcadas en su propio avance entre la primera evaluación (mayo-Junio) y la segunda (noviembre-diciembre), en cada grupo y por tipo de contexto. En el gráfico 1 se describe que los directivos y profesores del contexto vulnerable (Comas) presentaron diferencias mayores a 50 puntos promedio, lo cual ha sido significativo en el contraste de ambos momentos $\left(t_{(108)}=-2571 ; p<.001\right)$, de igual modo, se han obtenido estas diferencias en la muestra de contextos medianamente vulnerables (Puente Piedra y Los Olivos) con más de 40 puntos promedio en similar comparación $\left(t_{(146)}=-1,675 ; p<.001\right)$. 
Competencias digitales en directivos y profesores aislados en el contexto de educación remota del año 2020

\section{Gráfico 1 \\ Promedios en la variable competencias digitales}

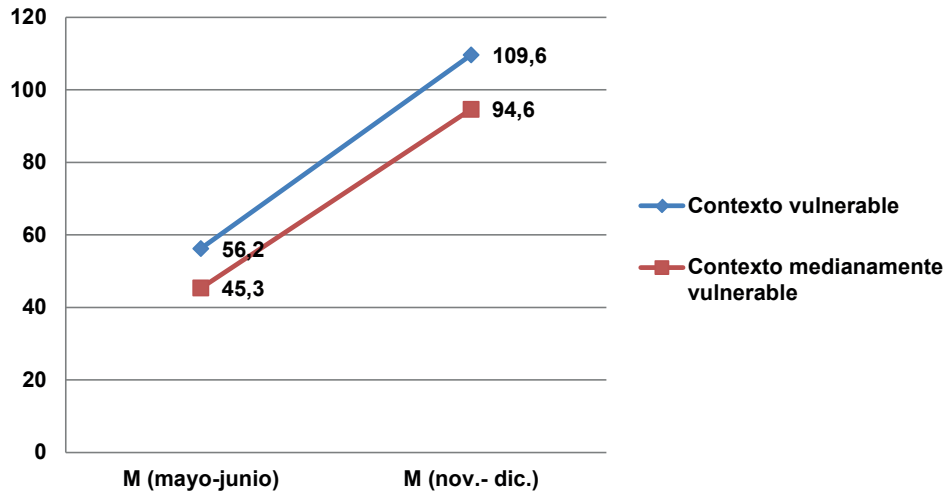

Fuente: Elaboración propia

En cuanto al gráfico 2, las habilidades desarrolladas de acuerdo al contexto, han sido más prominentes en las dimensiones comunicación y colaboración con más de 10 puntos porcentuales en los docentes que laboraban en contextos vulnerables. Los individuos del contexto medianamente vulnerable han presentado incremento en esta dimensión, pero en menor medida.

\section{Gráfico 2 \\ Promedios en las dimensión conocimiento y uso de la información}

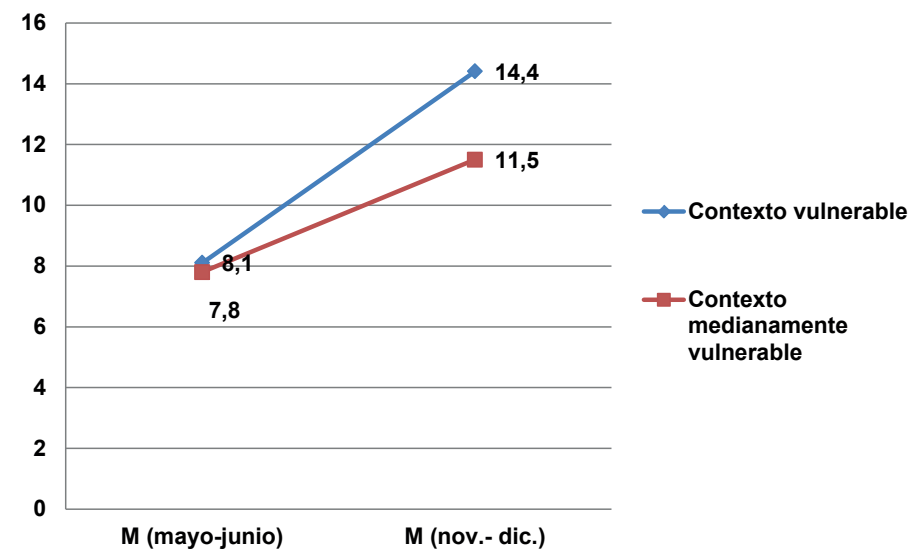

Fuente: Elaboración propia 
Respecto al gráfico 3 , sobre la dimensión comunicación y colaboración, se describen promedios homólogos respecto al inicio de la pandemia. Al finalizar, los docentes del contexto vulnerable reportaron en más de 5 puntos promedio, demostrar mejores habilidades para comunicarse, a diferencia de los provenientes de los profesores de contextos mediantemente vulnerables, quienes han demostrado menores niveles de desarrollo en esta habilidad.

\section{Gráfico3}

Promedios en las dimensión comunicación y colaboración.

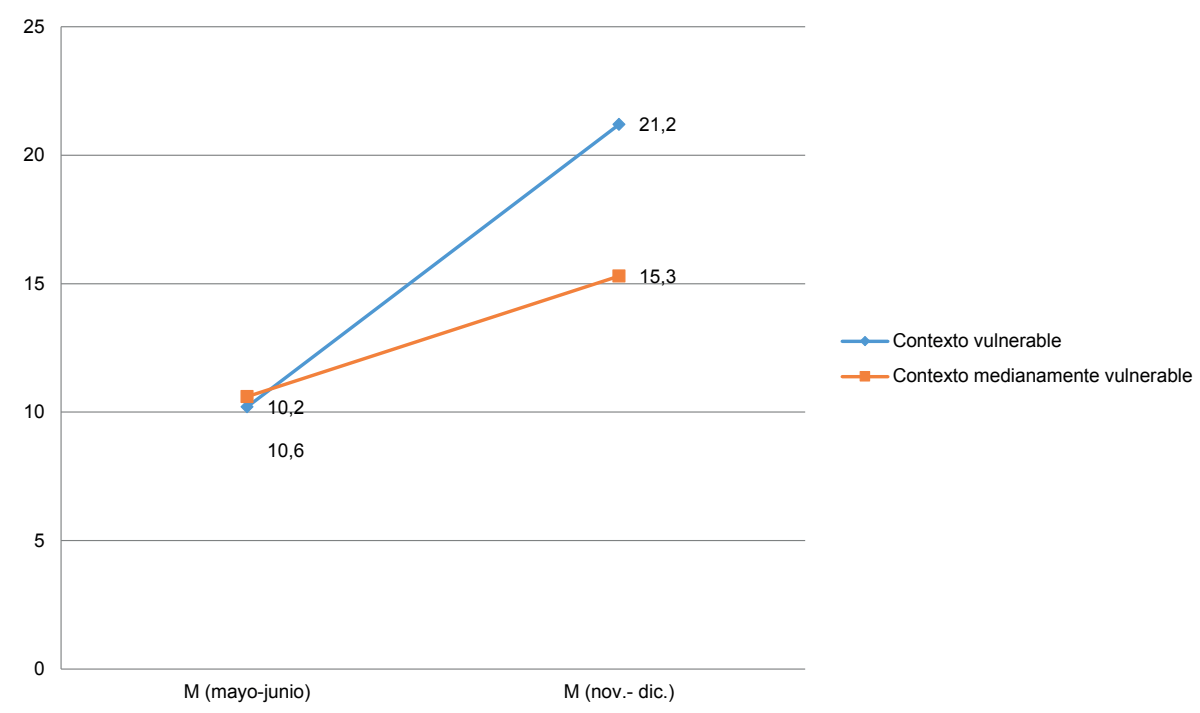

Fuente: Elaboración propia

En el gráfico 4, se manifiestan diferencias imperceptibles entre los docentes de los dos contextos evaluados, respecto a su habilidad para crear contenidos digitales. Al finalizar el año 2020, los del contexto vulnerable han demostrado haber desarrollado las competencias para elaborar recursos virtuales, físicos o mixtos para la didáctica en la enseñanza, así como también para utilizar tecnologías básicas para el desarrollo de sus clases virtuales. 
Competencias digitales en directivos y profesores aislados en el contexto de educación remota del año 2020

\section{Gráfico 4 \\ Promedios en la dimensión crear contenido digital}

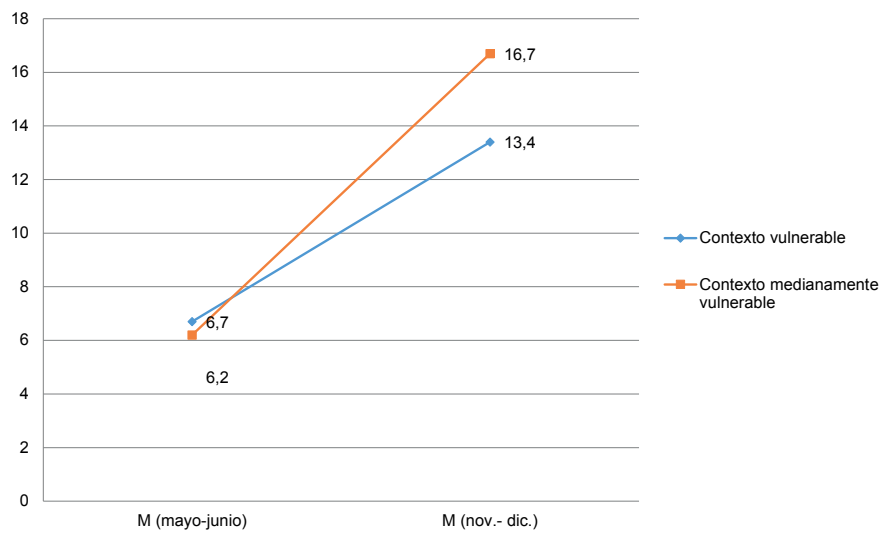

Fuente: Elaboración propia

En función del gráfico 5 sobre las acciones para generar seguridad digital en la enseñanza virtual, si se pueden aducir diferencias en el promedio de capacidades de los docentes al iniciar la pandemia con más de 1 punto promedio favorable al grupo de individuos del contexto vulnerable. Aunque en el desarrollo de la pandemia estas capacidades se doblaron para ambos grupos. Las diferencias fueron mínimas en el desarrollo de esta competencia al finalizar el año 2020.

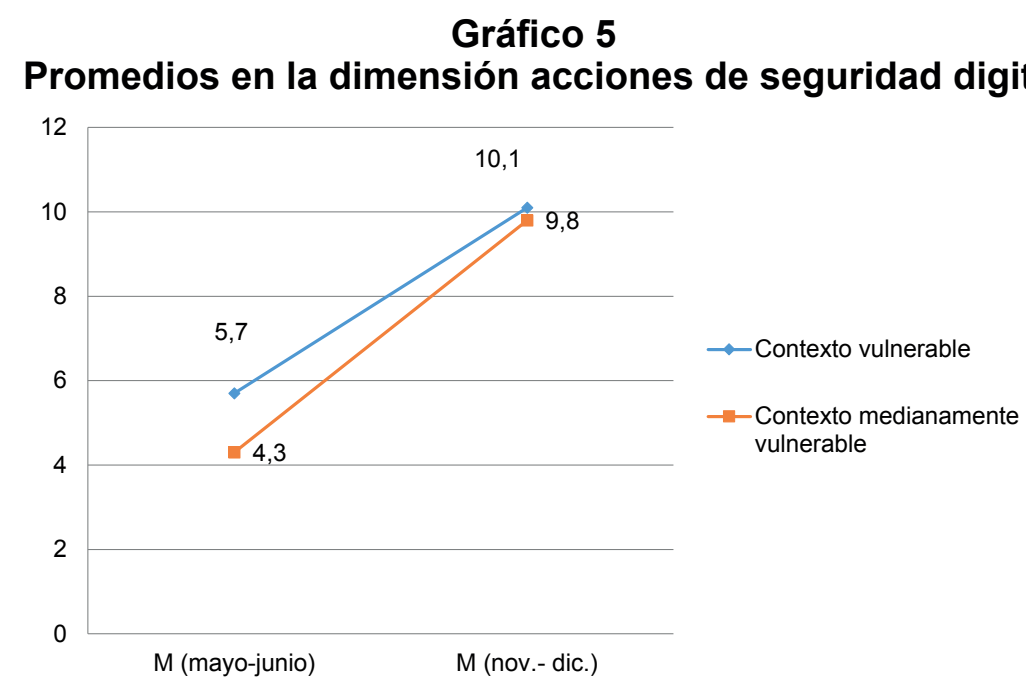

Fuente: Elaboración propia

634 - Esta obra está bajo una licencia de Creative Commons Atribución-Compartirlgual 4.0 Internacional (CC BY-SA 4.0) https://creativecommons.org/licenses/by-sa/4.0/deed.es https://www.produccioncientificaluz.org/index.php/rvg 
Del gráfico 6, se desligan igualdades en el uso de la habilidad resolver problemas en entornos digitales en ambos grupos, con diferencias menores a 1 punto promedio. En cambio, el incremento fue de 5 puntos porcentuales para el grupo del contexto vulnerable y de 4 para el grupo de docentes del contexto medianamente vulnerable. De igual modo, las diferencias en el desarrollo de esta capacidad fueron muy mínimas y poco representativas.

\section{Gráfico 6 \\ Promedios en la dimensión resolver problemas en entornos}

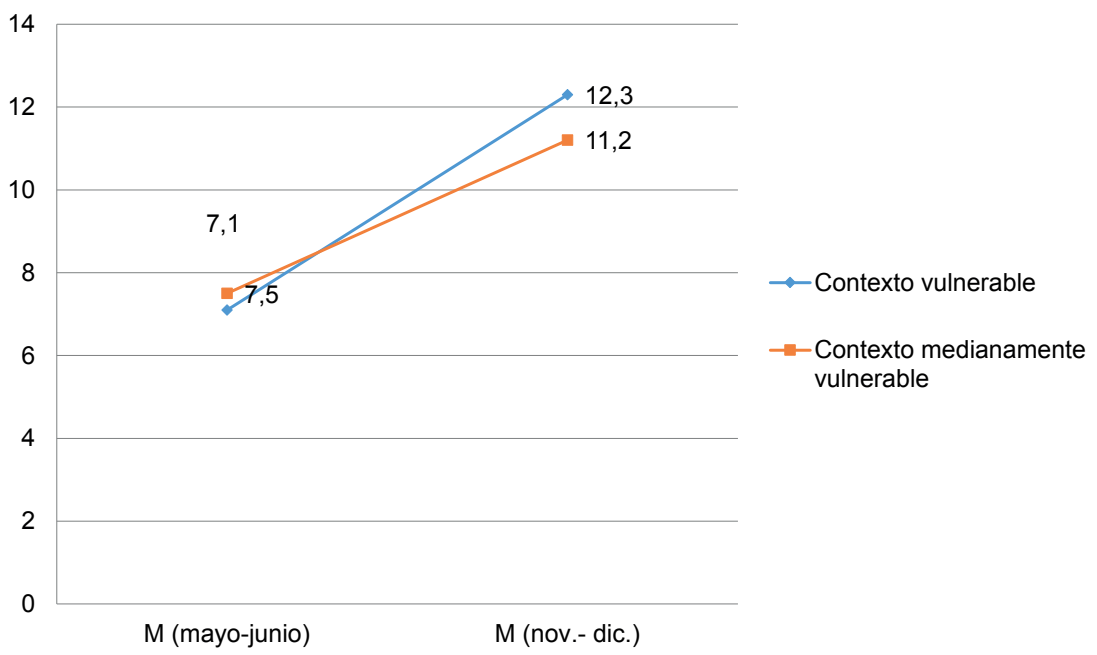

Fuente: Elaboración propia

Los objetivos del estudio han permitido realizar análisis por grupos individuales, también se puede apreciar que en la muestra del contexto vulnerable se presentó mayor puntuación que los del contexto de vulnerabilidad media. Aunque en este caso, nacen incertidumbres respecto a la determinación de estos resultados, debido las diferencias en el promedio de cada grupo lo cual impide hacer una comparación de muestras independientes. Sin embargo, se puede aducir que durante el transcurso de la pandemia ha prevalecido mayor crecimiento en el contexto vulnerable, a pesar de que en ambos grupos se ha dado este aumento.

\subsection{Competencias digitales por tipo de muestra}

De acuerdo a la tabla 2, se identifica mejor puntuación en competencias digitales en la muestra de directivos desde el inicio de la pandemia y su proceso (noviembre y diciembre) en 
Competencias digitales en directivos y profesores aislados en el contexto de educación remota del año 2020

el año 2020, tanto en el acumulado de habilidades como en cada una de ellas, este incremento fue significativo en cada contraste (t-comp.dig. ${ }_{(74)}=-1,204$; $t$-conoc.uso (73) $=-1,502 ; \quad t$-com.col. ${ }_{(76)}$ $=-1,225 ;$ t-con.dig. ${ }_{(71)}=-1,834 ; t$-seg. dig. $_{(69)}=-1,304$; t-res.prob. $._{(73)}=-1,508$; $p<.001)$. También se pudo observar mejor progreso entre los promedios obtenidos de la muestra de profesores en el mismo lapso. Un análisis posterior de estos datos reportó índices significativos (t-comp.dig. $_{{ }_{(198)}}=-2,383 ;$ t-conoc. uso $_{(196)}$ $=-2,272 ; t$-com.col. ${ }_{(189)}=-2,384 ; t$-con. dig $_{(197)}=-2,301 ;$ t-seg.dig. $\cdot_{(191)}=-2,101$; t-res.prob. $\left.{ }_{(197}=-2,155 ; p<.001\right)$.

\section{Tabla 2}

\section{Promedios sobre competencias digitales en directivos y profesores respecto al momento de evaluación del año 2020}

\begin{tabular}{ccccc}
\hline \multirow{2}{*}{ Variables y dimensiones } & \multicolumn{2}{c}{ Promedios (directivos) } & \multicolumn{2}{c}{ Promedios (profesores) } \\
\cline { 2 - 5 } & $\begin{array}{c}M[\text { ma- } \\
\text { yo-Junio] }\end{array}$ & $\begin{array}{c}M \text { [nov.- } \\
\text { dic.] }\end{array}$ & $\begin{array}{c}M \text { [ma- } \\
\text { yo-Junio] }\end{array}$ & $\begin{array}{c}M \text { [nov.- } \\
\text { dic.] }\end{array}$ \\
\hline Comp. Digitales [variable] & 43,2 & 81,2 & 56,7 & 102,3 \\
\hline $\begin{array}{c}\text { Conocimiento y uso de la } \\
\text { información }\end{array}$ & 8,2 & 15,6 & 7,5 & 14,3 \\
\hline Comunicación y colaboración & 9,2 & 18,3 & 8,4 & 16,7 \\
\hline Crear contenido digital & 5,6 & 13,2 & 3,4 & 12,1 \\
\hline Acciones de seguridad digital & 3,4 & 7,8 & 4,5 & 8,5 \\
\hline Resolver problemas en en- & 5,1 & 10,3 & 6,4 & 12,4 \\
\hline tornos & & & & \\
\hline
\end{tabular}

Fuente: Elaboración propia

Nota: $\mathrm{M}=$ promedio.

Del gráfico 7 , se denota que hubo mayor desarrollo de las competencias digitales en la muestra de profesores, siendo que más del $60 \%$ del total desarrollaron estas habilidades de forma avanzada. La diferencia también se evidencia en el $2 \%$ de directivos que superan al grupo de docentes quienes desarrollaron competencias digitales ínfimas. 


\section{Gráfico 7 \\ Competencias digitales de acuerdo al tipo de muestra}

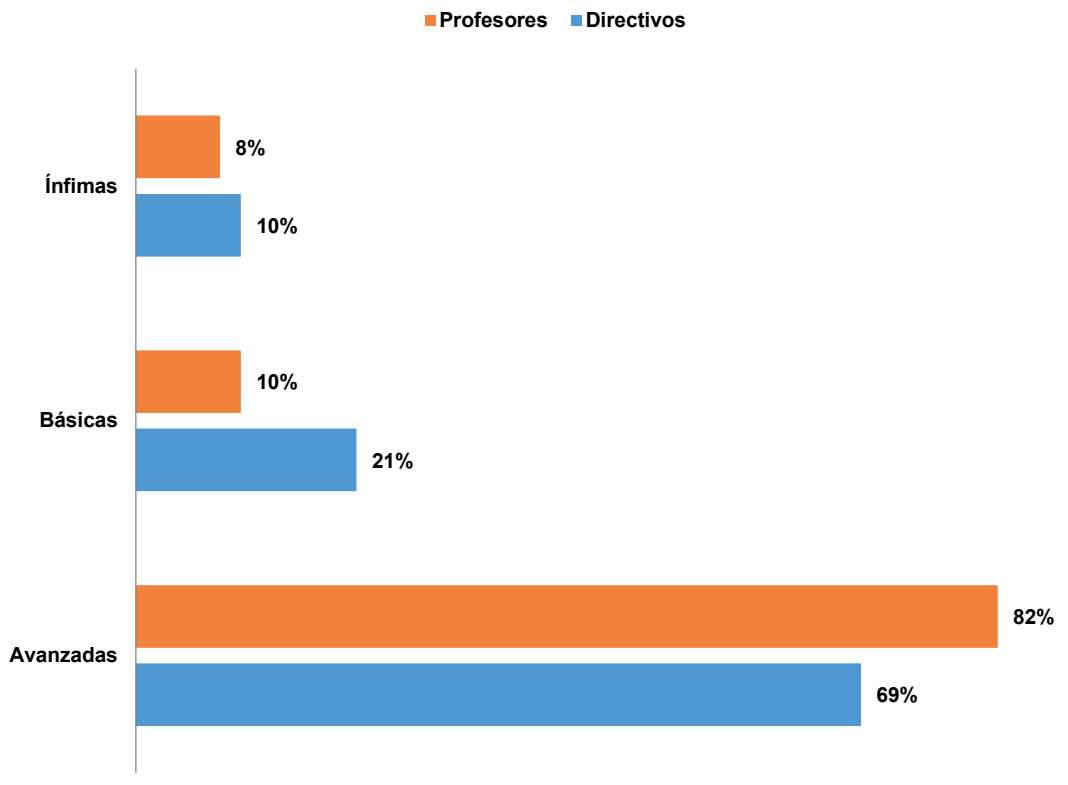

Fuente: Elaboración propia

En cuanto a la dimensión conocimiento y uso de la información más del $15 \%$ de los docentes encuestados lograron desarrollar esta competencia con carácter de avanzado. Más del $20 \%$ del total de muestra de directivos demostró nivel ínfimo al finalizar el año 2020. En cuanto a la dimensión comunicación y colaboración, alrededor del $80 \%$ de directivos y profesores lograron adquirir estas capacidades, demostrando ser de alto nivel o avanzadas. Por otro lado, menos del $5 \%$ de docentes demostraron nivel ínfimo, pero casi el $15 \%$ de directivos no lograron desarrollar estas capacidades en el nivel básico o avanzado.

Para la dimensión creación del contenido digital, surge una diferencia mayor al $10 \%$ entre docentes y directivos en relación al nivel avanzado, siendo mayor la muestra de directivos en donde se observó mejor desarrollo de esta dimensión. Es decir, que los directivos lograron en mayor número la capacidad para crear contenidos virtuales para la enseñanza o exposición. En tanto que el $20 \%$ de profesores ha demostrado nivel ínfimo de esta dimensión, menos del 5 \% llegó a ese nivel en el grupo de directivos.

Respecto a la seguridad digital, el avance en directivos y docentes fue menor al $70 \%$ del total de sujetos, por cuanto, este grupo ha logrado tomar en cuenta y advertir los mecanismos 
para la protección en el uso de entornos virtuales, la capacidad de expresar información privada y utilizarla de forma adecuada en redes sociales. Por otro lado, un promedio del $15 \%$ de directivos y docentes no logró desarrollar esta capacidad de forma positiva, pues se encontraron en nivel ínfimo en esta competencia.

Finalmente, Casi el $80 \%$ de los docentes y directivos han desarrollado la capacidad de resolver problemas en entornos virtuales, mientras que el 10 $\%$ de profesores y el $8 \%$ de directivos presentaron niveles ínfimos del desarrollo de esta competencia, por cuanto, este grupo tendría problemas para lograr plantear soluciones ante cualquier dificultad de enseñanza, desarrollar métodos que faciliten los aprendizajes en grupos estudiantiles masivos, y aplicar estrategias de enseñanza con distintos dispositivos tecnológicos.

\section{Discusión en el contexto de educación remota}

De acuerdo al objetivo del estudio competencias digitales de directivos y profesores de las instituciones educativas públicas y privadas de tres distritos de Lima, a través de la descripción de promedios y niveles de competencias digitales en la muestra, estos presentaron promedios que demostraron más desarrollo de sus habilidades desde la primera evaluación de mayo-Junio y noviembre-diciembre. Esto ha demostrado inicialmente que distintas necesidades pueden haber tenido efectos generados en la educación remota desde el mes de marzo.

Estas exigencias emergentes provocaron en los individuos el aprendizaje y búsqueda de la información, destacando la habilidad para investigar como un apoyo al desarrollo de las competencias digitales, es decir, los docentes y directivos se han adaptado al sistema de educación virtual (remota) obedeciendo al sentido de supervivencia educativa y social. La experiencia pandémica ha servido para comunicarse más y para generar espacios para aprender en la interacción virtual, ya sea académica o emocional.

Esto último demuestra la falta de acercamiento del currículo a la vida digital de los receptores [estudiantes], tal como lo han insinuado los planteamientos de Tomczyk (2020), sobre la comunicación segura en sociedades altamente interactivas, más ahora, el contexto pandémico ha exigido aplicar este concepto hacia una práctica profunda en la escuela, asimismo, los individuos de esta muestra se han adaptado a los efectos del aislamiento, por lo que han utilizado más vías, fuentes de información, lo cual ha exigido que desarrollen la capacidad de uso en los profesores y directores, siendo esta la mediación para generar hábitos culturales e intercambio de conocimiento, como se ha reportado en otros estudios (Choudrie et al, 2018). Ante el escenario de educación remota, esta capacidad y la de comunicación han sido esenciales para implementar alguna cultura digital en los contextos más necesitados.

Específicamente, el análisis de las competencias digitales de los docentes y los directivos, se hallaron mejoras esenciales en el total de las competencias de los estos, siendo duplicadas en cuanto a su promedio. No obstante, los docentes han representado mejores puntuaciones promedio de incremento en la habilidad para generar contenidos digitales y en la capacidad para resolver problemas. 
Aunque en esta última, los directivos les igualaron en su desarrollo. Todo esto configuran dos tipos de competencia que usualmente se desarrollan en la presencialidad como el uso de recursos o materiales pedagógicos y la toma de decisiones, en ambos casos (aunque haya sido obligatorio), las necesidades del sistema de educación remota ha hecho vislumbrar todo el repertorio de adecuación de los docentes a un sistema de educación digital, de forma que utilicen su creatividad aplicada al uso de herramientas 3.0 y 4.0 .

Igualmente, los individuos de la muestra han aprendido a decidir sobre las acciones de enseñanza, el tiempo de consumo de sus clases o gestiones directivas, en tanto que, se han generado oportunidades para escuchar, analizar distintas situaciones de aprendizaje virtual y ajustar sus tiempos para resolver la educación ante la emergencia sanitaria en Perú.

Estas evidencias se corresponden con los planteamientos de los autores que han declarado la necesidad formativa de la educación virtual con estrategias colaborativas (Garzón et al, 2020; Engen, 2019), como base de la superación en comunidades digitales que cada vez se integran a los espacios de desarrollo social en entornos virtuales. En Perú, los directivos y docentes han tomado su experiencia durante el año lectivo para utilizar recursos tecnológicos y virtuales ante la necesidad de olvidar los recursos presenciales.

Adicional, la resolución de problemas ha implicado otras capacidades como la resiliencia, la toma de decisiones y el autocontrol, las cuales están implícitas en la interacción de aprendizaje en los espacios virtuales de enseñanza. Aristizabal \& Cruz (2018), ya han plasmado el uso de dispositivos digitales como una herramienta de "salvación" de la educación virtual, sobre ello, muchos de los profesores y directivos han recurrido únicamente a la telefonía móvil y a sus aplicativos de contacto para solucionar diversos problemas como el enlace por medio de Internet y la falta de recursos tecnológicos.

Los resultados descriptivos de la tercera parte del análisis, reportaron el crecimiento en los niveles de las habilidades digitales para comunicarse y aprender colaborando, el desarrollo ha sido en mayor proporción en profesores que en directivos, lo cual ha demostrado que al culminar el año 2020 [noviembrediciembre] los individuos evaluados han realizado diferentes actividades de gestión que les ha exigido establecer nexos o vías para transferir información, más del $70 \%$ ha interactuado con sus propios recursos.

Al respecto, otras evidencias han declarado que los sujetos que se interrelacionan más suelen adquirir competencias para innovar, investigar y actualizarse, si los propósitos de aprendizaje tienen alguna orientación de desarrollo personal (Andía et al, 2020; Aristizabal \& Cruz, 2018). La necesidad de establecer diversos apoyos para la comunicación virtual ha exigido que, a la par, los participantes generen otros recursos de enseñanza más factibles, para suplir la falta de herramientas de interacción virtual que sean mucho más didácticas.

Sobre lo señalado, según Engen (2020) se podría criticar la capacitación y actualización de los agentes educativos para eventos emergentes, sin lugar a dudas, es necesario discernir que estas habilidades se han desarrollado y adaptado en la educación remota, de acuerdo a las exigencias del 
aislamiento social, sin estar acorde a las habilidades que constantemente desarrollan los individuos como parte de su autoaprendizaje.

Esto se ha notado con más claridad en la dimensión acciones de seguridad digital. Casi el $40 \%$ de cada muestra ha presentado problemas para adquirir habilidades avanzadas, considerando que la evaluación integra el conocimiento de recursos 3.0, esto demuestra que los directivos y profesores puede realizar actividades pedagógicas con ciertos riesgos en el uso de los espacios virtuales de aprendizaje, redes sociales y páginas web; en algunos de los casos no utilizaban algunos de estos, sin ser conscientes de su utilidad para aprendizajes colaborativos (Engen, 2020; Fallon, 2020, Garzón et al, 2020, Spante et al, 2018).

Entonces, cabe otorgar mayor pertinencia al liderazgo digital del currículo, su adaptación a las características de consumo estudiantil, al uso del Internet que hacen para mantenerse interrelacionados; con el fin de incluirlos en distintas comunidades digitales evitando los peligros intrínsecos que contrae el uso de los espacios virtuales.

\section{Conclusiones}

Ante los resultados encontrados, las competencias se han desarrollado con mejores puntuaciones en los profesores con necesidades económicas y sociales [contexto vulnerable], tanto en las sociedades en que conviven, como en las instituciones en que laboran. Respecto a ello, es importante establecer que los profesores que ejecutan la tarea docente en aislamiento son mejores generadores de estrategias para la comunicación, la resolución de problemas y la capacidad de utilizar la información para el aprendizaje tanto como para comunicar nuevos conocimientos en el trabajo académico.

Las competencias digitales se han desarrollado más en los profesores y directivos de la muestra evaluada, siendo de mayor nivel en el contexto vulnerable (distrito de Comas) a diferencia de los profesores que han ejercido la docencia con alumnos de contextos medianamente vulnerables, las cuales se sustentan en diferencias significativas favorables entre las evaluaciones realizadas. En esta comparación, los individuos del contexto vulnerable han presentado mayor puntuación y mejora en dimensiones como el conocimiento y uso de la información, comunicación y colaboración, y resolver problemas en entornos digitales. Las acciones de seguridad digital es la habilidad de menor arraigo en la muestra respecto al contexto.

Desde los resultados por tipo de individuo, los profesores han desarrollado más habilidades que los directivos entre los meses de mayo y diciembre de 2020 , por lo que el promedio de crecimiento de sus habilidades se ha concentrado más para comunicarse, enseñar de forma colaborativa, utilizar la información y generar recursos digitales. En cuanto a la dimensión acciones de seguridad digital, ambas muestras la desarrollan en menor nivel; sin embargo, los directivos utilizan menos habilidades para asegurar los procesos de aprendizaje en entornos virtuales.

Se detectó alto nivel de las capacidades para comunicarse en los profesores y directores, tanto como para utilizar información en la enseñanza, siendo estas habilidades de tipo avanzadas en más de la mitad de los evaluados. En cuanto al uso de recursos 
digitales, se encontraron promedios similares en ambas muestras, con mayor promedio en la muestra de profesores, quienes llegaron en porcentaje considerable a nivel avanzado. Los niveles en la dimensión acciones de seguridad digital alrededor del 40 $\%$ de cada muestra ha demostrado capacidades básicas o ínfimas.

\section{Referencias bibliográficas}

Acevedo-Duque, Á., Argüello, A.J., Pineda, B.G., Turcios, P.W. (2020). Competencias del docente en educación online en tiempo de COVID-19: Universidades Publicas de Honduras. Revista de Ciencias Sociales, 26(2), 206-224. https://doi. org/10.31876/rcs.v26i0.34123

Allueva, P. \& Alejandre, M. (2019). Enfoques $y$ experiencias de innovación educativa con TIC en educación superior. Prensas de la Universidad de Zaragoza.

Andía, L.A., Campión, R.S., \& Sota, J.M. (2020). ¿Estamos técnicamente preparados para el flippedclassroom? Un análisis de las competencias digitales de los profesores en España. Contextos educativos. Revista de Educación, 25, 275-311. https://doi.org/10.18172/con.4218

Aristizabal, P. \& Cruz, E. (2018). Development of digital competence in the initial teacher education of early childhood education. Pixel-bitRevista de medios y educacion, 52, 97-110. https://doi.org/10.12795/ pixelbit.2018.i52.07

Brauer, S. (2019). Digital Open BadgeDriven Learning-Competencebased Professional Development for Vocational Teachers. Acta Universitatis Lapponiensis 380, University of Lapland Printing Centre, Rovaniemi, 380.https://lauda.ulapland.fi/ bitstream/handle/10024/63609/ Brauer Sanna ActaE 247pdfA. pdf? sequence $=1$ \&isAllowed $=y$

Brolpito, A. (2018). Digital Skills and Competence, and Digital and Online Learning. European Training Foundation.https://eufordigital.eu/ library/digital-skills-and-competenceand-digital-and-online-learning/

Choudrie, J., Pheeraphuttharangkoon, S., \& Davari, S. (2018). The digital divide and older adult population adoption, use and diffusion of mobile phones: A Quantitative Study. Information Systems Frontiers, 21(98), 1-29.https://doi.org/10.1007/ s10796-018-9875-2

Engen, B. (2019). Understanding social and cultural aspects of teachers' digital competencies. Comunicar, 27(61), 9-19.https://doi.org/10.3916/ C61-2019-01

Fallon, G. (2020). From digital literacy to digital competence: the teacher digital competency (TDC) framework. Educational Technology Research and Development, 68, 2449-2472. https://doi.org/10.1007/s11423-02009767-4

Gobierno del Perú (2020). Decreto Supremo $N^{\circ}$ 046-2020-PCM.https:// www.gob.pe/institucion/pcm/normaslegales/462244-046-2020-pcm

Garzón, A., Sola, M., Ortega, M., Marín, M. \& Gómez, G. (2020). Teacher Training in Lifelong Learning-The Importance of Digital Competence in the Encouragement of Teaching Innovation. SustainabilityJournal, 12(7), 1 - 13.https://doi.org/10.3390/ su12072852

Girón-Escudero, V., Cózar-Gutiérrez, R., \& González-Calero, J.A. (2019). Análisis de la autopercepción sobre el nivel de competencia digital 
Holguin-Alvarez, Jhon; Apaza-Quispe, Juan; Ruiz Salazar, Jenny Maria y

Picoy Gonzales, Juan Antonio

Competencias digitales en directivos y profesores aislados en el contexto de educación remota del año 2020

docente en la formación inicial de maestros/as. Revista Electrónica Interuniversitaria de Formación del Profesorado, 22(3), 193-218.https:// doi.org/10.6018/reifop.373421

Holguin-Alvarez, J., Villena-Guerrero, M., Soto-Hidalgo, C., \& PanduroRamírez, J. (2020). Competencias digitales, liderazgo distribuido $y$ resiliencia docente en contextos de pandemia. Revista Venezolana de Gerencia, 25(4), 38-53. https:// produccioncientificaluz.org/index. php/rvg/article/view/35175

Instituto Nacional de Estadística e Informática del Perú (INEI, 2020). Más de medio millón de maestros en el Perú celebran su día.http://m. inei.gob.pe/prensa/noticias/mas-demedio-millon-de-maestros-en-elperu-celebran-su-dia-9833/

Instituto Nacional de Estadística e Informática (INEI, 2019). Estadísticas de las Tecnologías de Información y Comunicación en los Hogares.https://www.inei.gob.pe/ media/MenuRecursivo/boletines/ ticdiciembre.pdf

Janssen, J., Stoyanov, S., Ferrari, A., Punie, Y., Pannekeet, K., \&Sloep, P. (2013). Experts' views on digital competence: Commonalities and differences. Computers \& Education, 68, 473-481.https://doi. org/10.1016/j.compedu.2013.06.008

Lasso, E.P., Munévar, P.A., Rivera, J.A., \& Sabogal, A. (2017). Estado del arte sobre la articulación de modelos enfoques y sistemas en educación virtual. Libros Universidad Nacional Abierta y a Distancia, 1-135. https:// hemeroteca.unad.edu.co/index.php/ book/article/view/1969

Levano-Francia, L., Sanchez, S., Guillén-Aparicio, P., Tello-Cabello, S., Herrera-Paico, S., \& CollantesInga, Z. (2019). Competencias digitales y educación. Propósitos y Representaciones, 7(2), 569-588. http://dx.doi.org/10.20511/pyr2019. v7n2.329

Ministerio de Educación del Perú (2020). Minedu suspende clases en universidades públicas $y$ privadas para evitar propagación del coronavirus. https://www. gob.pe/institucion/minedu/ noticias/108801-minedu-suspendeclases-en-universidades-publicasy-privadas-para-evitar-propagaciondel-coronavirus

Ministerio de Salud (Minsa 2020). Casos confirmadosporCOVID-19ascienden a 13489 en el Perú (Comunicado N . 68). https://www.gob.pe/institucion/ minsa/noticias/126083-minsa-casosconfirmados-por-coronavirus-covid19-ascienden-a-13-489-en-el-perucomunicado-n-68

Ministerio de Salud (Minsa 2020). Casos confirmados por COVID-19 ascienden a 1017199 en el Perú (Comunicado $\mathrm{N}^{\circ}$. 375). https:// www.gob.pe/institucion/minsal noticias/322964-minsa-casosconfirmados-por-coronavirus-covid19-ascienden-a-1-017-199-en-elperu-comunicado-n-375

Ruíz-Velazco, S. \& Barcena, L. (2019). Edutecnología y Aprendizaje 4.0. SOMECE.

Sandí, J.C. \& Sanz, C.V. (2020). Juegos serios para potenciar la adquisición de competencias digitales en la formación del profesorado. Revista Educación, 44(1), 2215-2644. http://dx.doi.org/10.15517/revedu. v44i1.37228

Siemens, G. (2014). Connectivism: A Learning Theory for the Digital Age. [elearnspace.org]. https://bit. Iy/2QjoRm4

Spante, M., Hashemi, S., Lundin, 
pp. 623-643

Revista Venezolana de Gerencia, Año 26 No. 94, 2021

M., \&Algers, A. (2018). Digital Competence and Digital Literacy in Higher Education Research: Systematic Review of Concept Use. Cogent Education Journal, 5 (1). 1-21.https://doi.org/10.1080/233118 $\underline{6 X .2018 .1519143}$

Teixeira, D. \& Martini, S.C. (2019). Conectivismo Pedagógico: novas formas de ensinar e aprender no século XXI. RevistaThema, 16(4), 1012-1025.https://doi.org/10.15536/ thema.V16.2019.1012-1025.1583

Tomczyk, L. (2019). Skills in the area of digital safety as a key component of digital literacy among teachers. Education and Information Technologies, 25, 471-486.https:// doi.org/10.1007/s10639-019-09980$\underline{6}$

Tourón, J., Martín, D., Navarro, E., Pradas, S., \& Îñigo, V. (2018). Validación de constructo de un instrumento para medir la competencia digital docente de los profesores (CDD). Revista Española de Pedagogía, 76(269), 25-54.https://doi.org/10.22550/ REP76-1-2018-02

World Bank (2019). Digital
Development. Washington DC.
https://www.worldbank.org/en/topic/
digitaldevelopment

